Questions vives

\section{Questions Vives}

Recherches en éducation

$N^{\circ} 25$ | 2016

L'activité des enseignants face au décrochage scolaire : Quelles difficultés et quelles mises en œuvre professionnelles?

\title{
Le collectif de travail au centre des dispositifs de prévention du décrochage des élèves en éducation physique et sportive
}

A work collective to prevent dropout in physical education lessons

\section{Jacques Méard}

\section{(2) OpenEdition \\ Journals}

\section{Édition électronique}

URL : http://journals.openedition.org/questionsvives/1871

DOI : 10.4000/questionsvives.1871

ISSN : $1775-433 X$

Éditeur

Université Aix-Marseille (AMU)

Édition imprimée

Date de publication : 7 juillet 2016

ISBN : 978-2-912643-49-0

ISSN : 1635-4079

Référence électronique

Jacques Méard, "Le collectif de travail au centre des dispositifs de prévention du décrochage des

élèves en éducation physique et sportive », Questions Vives [En ligne], № 25 | 2016, mis en ligne le 05 septembre 2016, consulté le 22 avril 2019. URL : http://journals.openedition.org/questionsvives/1871 ; DOI : 10.4000/questionsvives. 1871

Ce document a été généré automatiquement le 22 avril 2019.

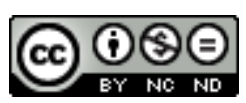

Questions Vives est mis à disposition selon les termes de la licence Creative Commons Attribution -

Pas d'Utilisation Commerciale - Pas de Modification 4.0 International. 


\title{
Le collectif de travail au centre des dispositifs de prévention du décrochage des élèves en éducation physique et sportive
}

\author{
A work collective to prevent dropout in physical education lessons
}

Jacques Méard

\section{Introduction}

1 Un récent dossier de l'Institut Français de l'Éducation (Thibert, 2013) rend compte de l'intérêt pour le décrochage scolaire (DS), enjeu majeur des politiques publiques en matière d'éducation (Blaya, 2013). Même si, paradoxalement, il n'y a jamais eu si peu de décrocheurs depuis que l'école républicaine existe (Bernard, 2011, p. 40), le DS devient le principal indicateur de la réussite et de l'échec des politiques d'éducation (Commission Européenne, 2000). Les sphères institutionnelles et professionnelles en France entretiennent cette préoccupation par une abondante littérature sur le sujet. Ainsi, Bruno, Méard et Walter (2014) identifient 22 textes institutionnels français prescrivant 24 dispositifs de lutte contre le DS en collège, dont neuf publiés après janvier 2011.

2 Concrètement, le DS se définit par l'abandon prématuré par un élève de son parcours scolaire. Mais cet abandon est souvent l'aboutissement d'un désengagement progressif, un "décrochage de l'intérieur» (Bonnery, 2007): des distractions épisodiques puis fréquentes, des échecs cumulés ou mal acceptés, de l'ennui, conduisant à un désinvestissement croissant, des absences perlées puis continues. Selon les résultats des travaux scientifiques, ce processus de désadhésion progressive au travail scolaire (Blaya \& Hayden, 2003) résulterait de l'influence croisée de plusieurs facteurs scolaires (Lee \& Burkham, 2003) et extrascolaires tels que les facteurs sociaux, économiques, familiaux (Cairns, Cairns \& Neckerman, 1989). 
3 Dans ce cadre, par la mise en place de programmes de prévention, l'école lutte contre le DS par une adaptation des cursus et accompagnements scolaires aux différents types d'élèves «à risque de décrochage » et par un développement de partenariats éducatifs, notamment avec les parents ou les structures de prise en charge des élèves en dehors de l'école (Tièche Christinat \& Gilles, 2013). Mais l'évaluation de ces programmes de prévention est souvent négligée (Blaya, 2010) ou conduit le plus souvent soit à l'impossibilité de mesurer leurs effets réels, soit au constat que ces programmes ont peu ou pas d'effet (Bruno, 2015).

4 La dimension décevante de ces évaluations trouve son origine dans l'impossibilité de distinguer clairement les facteurs scolaires des facteurs extrascolaires du DS tant ils apparaissent imbriqués dans l'activité des acteurs en situation (Brown \& Rodriguez, 2009). Plusieurs auteurs mettent aussi en évidence que les programmes de prévention du décrochage sont toujours déclinés localement de façon spécifique. En d'autres termes, les enseignants modifient toujours le programme qu'ils sont supposés appliquer (Dusenbury, Brannigan, Hansen, Walsh \& Falco, 2005). Dans le contexte francophone, cette composante locale est reprise dans des approches québécoises telle que la "Stratégie d'intervention Agir autrement » (SIAA) (Bélanger, Bowen, Cartier, Desbiens, MontésinosGelet \& Turcotte, 2012) et par les recherches françaises d'inspiration ergonomique montrant que les travailleurs de l'éducation (comme les autres) n'exécutent jamais complètement ni exactement une prescription, quand bien même ils y adhèrent (Durand, 1996 ; Félix \& Saujat, 2008), notamment pour prévenir le DS (Flavier \& Moussay, 2014 ; Méard, 2014).

5 Autrement dit, même si les recherches convergent sur un certain nombre de principes concernant la prévention du DS (inscription de dispositifs sur le long terme, délimitation d'objectifs précis, évaluation des résultats des actions menées, implication d'équipes éducatives cohérentes) (Blaya, 2010), la compréhension fine de ce qui amène un élève à se désengager du travail scolaire et les effets précis des dispositifs et programmes spécifiques sur ces pratiques scolaires réelles ne sont pas identifiés (Houlfort \& Sauvé, 2010). Ce constat pousse à chercher des pistes de compréhension dans la classe ellemême, dans les interactions d'enseignement-apprentissage (Blaya, 2010).

Or, en classe, le DS est rapporté à deux grandes voies de compréhension : premièrement au "sens" que l'élève attribue ou non au travail scolaire, ce qui interroge les opportunités qu'offre le «jeu didactique» mis en œuvre par l'enseignant avec l'apprenant (Sensevy \& Mercier, 2007) et plus globalement la finalisation des contenus d'enseignement en direction de celui-ci. D'autres travaux englobent dans cette problématique du « sens » la part non disciplinaire des apprentissages qui semblent jouer un rôle important dans le désengagement. Ils partent du constat que le travail scolaire est énoncé sous forme de règles sociales (être à l'heure, ne parler qu'après autorisation, ne pas se moquer des camarades, etc.) et disciplinaires (les contenus scolaires prennent aussi le format de règles énoncées en classe) (Méard, Bertone \& Flavier, 2008). L'adolescent investit ces prescriptions d'une signification ou s'y soumet sans comprendre, les négocie ou les enfreint selon une dynamique peu prévisible (Vors \& Gal-Petitfaux, 2009 ; Guérin, Pasco \& Riff, 2008). Dans cette perspective, le DS est compris au travers des interactions avec l'enseignant prescripteur du travail (la recherche d'une co-construction de sens) mais aussi des interactions entre pairs qui peuvent avoir des effets sur le réengagement scolaire chez des élèves "à risque »: amitiés, hostilités, harcèlement (Brown, 2010), moqueries (Thornberg, 2010). Plusieurs travaux pointent par exemple que la comparaison 
entre élèves dans une classe impacte négativement la persistance de l'engagement dans le travail scolaire (Thijs, Verkuyten \& Helmond, 2010).

Deuxièmement, le DS est aussi mis en lien en classe avec les difficultés rencontrées par les élèves dans les apprentissages. À ce niveau, Rumberger (2011) pointe des liens entre DS et difficultés scolaires. Selon Bautier (2002), le désengagement progressif de l'élève se rapporte à une conjonction de causes qui produisent de l'échec: des malentendus croisés (entre enseignant et élèves) à propos des attentes en termes d'acquisitions, un niveau insuffisant d'exigence au niveau des apprentissages, une non reconnaissance de difficultés précoces d'apprentissage qui conduit à méconnaître des processus d'abandon et à placer l'élève dans une situation de "non retour ». Dans cette optique, on considère l'élève qui décroche d'abord comme celui qui vit une constance d'échec, souvent caché, dans les apprentissages (voir aussi Bonnery, 2007). Par exemple, on pense au collégien qui accumule les déficits instrumentaux et cognitifs en primaire, déficits compensés par un soutien socio-affectif de la classe, mais qui « explose » quelques semaines après l'entrée au collège, face au niveau d'exigence et aux types d'interactions moins soutenants de la part des enseignants. Dans ces cas, on se rend compte que la mise au jour brutale de difficultés scolaires apparaît dans certaines disciplines, telles que les mathématiques et la langue première, qui ont dès lors une fonction prédictive concernant le DS (Deslandes \& Lafortune, 2001 ; Lessard, Fortin, Royer, Potvin, Marcotte \& Joly, 2007).

Dans cet article, nous voulons aborder les questions de la prévention du décrochage en collège dans une discipline scolaire qui est peu prédictive du DS : l'éducation physique et sportive (EPS). D'un poids peu important dans les décisions d'orientation, elle présente également un certain nombre de particularités qui la rendent singulière. D'abord, de nombreux élèves ont acquis des savoirs dans les activités physiques et sportives en dehors de l'école (en milieu associatif), parfois de façon massive. En outre, les difficultés d'apprentissage y sont visibles de tous : un collégien qui ne parvient pas à réaliser une roue en gymnastique chemine aux yeux de tous selon un processus d'apprentissage fait d'erreurs, de chutes, de postures parfois ridicules. Par ailleurs, en EPS, les élèves acquièrent des savoirs à propos de pratiques sociales (sports et danses) qui suscitent des émotions parfois fortes, de l'enthousiasme ou au contraire des rejets, de la peur. Ces trois particularités déclinent la problématique des difficultés d'apprentissage mais aussi celle du sens de façon accentuée. C'est la première raison pour laquelle il nous semble intéressant d'investiguer l'activité de prévention du DS dans cette discipline. De plus, en EPS, il existe un type de décrochage spécifique : la " dispense ", c'est-à-dire la possibilité pour l'élève de se soustraire à l'obligation de présence ou de participation en invoquant une inaptitude physique réelle ou simulée (Bergamaschi, 2014). Le fait que cette dispense soit majoritairement féminine (Mottet, Meraite \& Cloes, 2007), alors que le DS dans les pays occidentaux est essentiellement masculin (Blaya, 2013) permet d'aborder la question du désengagement de l'élève dans un contexte inhabituel. Enfin, comme le soulignent Bergamaschi et Méard (2012), la particularité de la prévention de la dispense d'EPS comme forme de DS embrasse des considérations sociales en classe et dans l'établissement, dans la mesure où ces inaptitudes temporaires sont déclarées souvent en début de leçon, du fait d'une indisposition, d'une blessure, ou à propos des absences relevées lors d'une leçon précédente; de même, l'élève peut ne pas participer mais reste présent en classe, sa non-participation peut être fondée sur une véritable inaptitude ou non. Autrement dit, il s'agit d'une forme de décrochage qui implique une gestion souvent 
dans l'urgence en classe et qui est l'objet de transactions entre professeur et élèves (Méard, Bertone \& Flavier, 2008).

9 L'étude présentée ici vise à mieux comprendre comment les enseignants d'EPS perçoivent et prennent en compte le processus de persistance ou de désengagement des élèves qui conduisent, dans certains cas, à des "dispenses". Par rapport aux mathématiques et langues premières, est-ce que dans cette discipline se déclinent de manière comparable les problématiques de «sens» et de "difficulté d'apprentissage», en lien avec le processus de DS?

\section{Cadre théorique}

Pour envisager ce questionnement, l'étude se fonde sur les concepts de la psychologie culturaliste. Dans ce cadre, Leontiev (1984) indexe les actions du sujet d'une part à des motifs renvoyant eux-mêmes à des mobiles vitaux (faire ceci / parce que), d'autre part à des opérations correspondant à la maîtrise d'instruments, de techniques (faire ceci / de cette façon). Ce postulat permet de lire l'activité des élèves et des enseignants en même temps en fonction du " sens » qu'ils accordent à leurs actions et de «l'efficience » de ces actions. Il autorise, du côté de l'élève, une analyse des deux grandes voies de compréhension du processus de DS évoquées précédemment (le déficit de sens et les difficultés d'apprentissage) et de caractériser les dynamiques d'interactions en classe, en rapport avec les motifs et opérations de l'enseignant. En même temps, on présuppose dans cette conception culturaliste que les acteurs au travail ne se contentent pas de réaliser le travail prescrit mais se développent grâce à des conflits intrapsychiques (Vygotski, 1934/1997), eux-mêmes issus de conflits interpsychiques (dans les interactions), de tensions dans les systèmes d'activité. Le travailleur en interaction oscille entre plusieurs possibles, négocie et « digère la prescription » en se l'appropriant et en la mettant «à sa main ", en la déclinant de manière personnelle grâce à un processus de renormalisation qui aboutit grâce à sa participation à un collectif de travail (Clot, 2008).

11 Mais ces deux premières générations de la psychologie culturaliste (Vygotski et Leontiev) ne permettent pas de prendre en compte le «système d'activités » dans lequel se déploient les actions de l'enseignant en classe. Or, concernant spécifiquement le sujet des dispenses d'EPS, les négociations entre élèves et enseignant en classe s'insèrent dans des usages d'établissement, un règlement. On peut avancer qu'en classe d'EPS, l'enseignant ne décide jamais seul. Pour cette raison, nous empruntons à la troisième génération des psychologues culturalistes l'idée que ces interactions se déploient dans des «systèmes d'activité » impliquant une division du travail (reposant sur des motifs partagés entre enseignants et élèves mais aussi entre enseignants, autres personnels de l'établissement et partenaires), des communautés (d'élèves, d'enseignants, ...) et des règles institutionnelles (Engeström, 2000). Ce modèle apporte donc des éclairages sur les dynamiques d'interactions en classe mais aussi au niveau des différents acteurs de l'établissement scolaire, afin de comprendre en quoi ce que fait l'acteur en classe, élève et enseignant, s'inscrit dans un "genre", dans des normes locales et dans un système réglementaire. Engeström pointe par exemple que les tensions sont liées au fait que les décisions dans les organisations de travail ne répondent pas forcément à l'organigramme affiché, que les pôles de décision sont changeants et interdépendants (ce qu'il définit comme "knotworking" ou " travail en nœuds »). De même, l'auteur met en évidence que les tâches réalisées par les acteurs débordent souvent de leur fiche de poste, empiètent sur 
les tâches des autres (ce qu'il définit comme "boundary crossing" ou "franchissement de frontière »). Ces concepts visent à comprendre les systèmes d'activités individuels à l'intérieur de l'organisation de la classe et de l'établissement.

\section{Méthode}

Dans le cadre d'un programme de recherche portant sur la prévention du décrochage scolaire, nous avons analysé selon une approche clinique 18 leçons menées par quatre professeurs d'EPS dans trois collèges du sud de la France. Ces trois établissements présentaient des caractéristiques proches : semi-urbains, de taille moyenne, obtenant des taux de réussite au Brevet et d'orientation très proches de la moyenne académique et des proportions d'orientation en fin de troisième comparables. Chacun comportait une équipe d'EPS de quatre à six enseignants. Les 18 analyses ont été menées dans six classes de quatrième et de troisième (13 à 16 ans). Les enseignants, deux hommes et deux femmes, étaient considérés comme chevronnés (âgés de 37 ans à 51 ans, plus de 10 années d'expérience en collège). Ces enseignants et les élèves des classes concernées ont participé volontairement à cette étude.

Les données sont d'une part issues de méthodes directes (observations, entretiens semidirectifs, entretiens informels avec les élèves et les enseignants) et indirectes (instructions au sosie, entretiens d'auto-confrontation). Le but était de comprendre les dynamiques d'engagement ou de désengagement des élèves en classe et les négociations relatives aux déclarations d'inaptitudes. La démarche comportait une dimension transformative en direction des participants, notamment grâce aux outils de méthode indirecte (Clot, 2008). Le recueil a consisté en de nombreuses données ethnographiques (32 heures, soit 13 leçons de $2 \mathrm{~h}$, deux leçons d'1h30 et trois leçons d'1h) : observations participantes, avec prises de note, interprétations à chaud, étayées par de courts entretiens informels (de 30 secondes à une minute) auprès des enseignants (36 entretiens) et des élèves ( 45 entretiens) en cours de leçon ou juste après la leçon. Au cours de cette première phase a débuté l'étude à proprement parler. Ainsi, ont été négociés progressivement avec les participants l'enregistrement audio (tablette numérique) de ces entretiens à chaud puis des entretiens semi-directifs (un avec chaque enseignant). Pour des raisons de faisabilité (compatibilité des emplois du temps, résistance de certaines familles à l'enregistrement vidéo), huit leçons ont alors été analysées en profondeur : enregistrement vidéo de la leçon, auto-confrontation simple avec l'enseignant concerné, interrogé à propos de ses actions en classe. Deux entretiens d'autoconfrontation croisée ont enfin été organisés et enregistrés.

Le traitement des données s'est développé en 4 étapes :

15 - d'abord le corpus entier des verbalisations au cours des huit leçons a été divisé en unités de transactions. Une transaction a été définie comme la séquence d'interaction incluant (a) le même objet (c'est-à-dire soit une seule règle énoncée, soit un enchaînement de règles liées les unes aux autres (par exemple, tu dois rester au bord du terrain (parce que) tu dois être prêt à entrer dans l'équipe à la prochaine rotation) et (b) les mêmes interlocuteurs ;

16 - ensuite les règles énoncées dans chaque unité de transaction ont été codées de façon standardisée, en dissociant (a) les prescriptions-proscriptions («tu dois», «tu ne dois pas»), qui pouvait prendre des formes nuancées («ce serait mieux que tu ...», « essaie 
de...») ou présentées comme une question («qu'est-ce que tu dois faire, là ?»); (b) les actions à faire (" faire ceci » "faire de cette façon »); et (c) les motifs (" pour », «parce que », «si tu ne fais pas ... »), qui étaient aussi parfois énoncées sous forme de questions ("pourquoi tu ne ferais pas de cette façon?»). Par exemple, l'action «vous vous souvenez, pour réussir une passe, on cherche d'abord les copains loin du défenseur » fut codée "vous devez (a) / chercher d'abord les copains loin des défenseurs (b) / pour réussir une passe (c) ». L'action "Sans crier ! La classe de M. X travaille à côté » fut codée « vous devez (a) / jouer sans crier (b) / pour laisser travailler la classe de M.X à côté (c) »;

17 - de plus, les actions des élèves désignés préalablement par l'enseignant comme «à risque décrochage » d'élèves furent observées et caractérisées en termes d'engagement (à partir des enregistrements) dans les unités de transaction et croisées avec les données d'entretiens pour mettre en évidence les dynamiques d'attribution de sens, en rapport avec les actions de l'enseignant (tableau de quatre volets, faisant succéder les unités de façon chronologique (Tableau 1). Volet 1 (actions et interactions verbales en classe), Volet 2 (règles codées), Volet 3 (entretien post-leçon avec l'enseignant) et Volet 4 (entretien avec l'élève);

18 - puis, chaque chercheur a interprété les leçons observées de façon indépendante en effectuant une triangulation des sources (observations, interactions verbales pendant des leçons et les entretiens) et en cherchant les tensions entre systèmes d'activité (Méard, 2008);

19 - enfin, une triangulation des chercheurs a permis d'analyser les types de tensions, les types de dynamiques de co-construction de sens, les scénarios-types en rapport avec le décrochage scolaire. Cette étape a abouti à des accords, la plupart du temps faciles à établir. En cas de désaccords, ils regardaient ensemble les éléments de la chronologie des transactions, les enregistrements des leçons et le discours de l'enseignant au cours de l'autoconfrontation (Méard, 2013).

\section{Résultats}

(Maude, Melissa, Hervé et Christophe sont les noms modifiés des quatre enseignants)

\subsection{La prévention du désengagement des élèves en classe}

21 L'analyse des résultats permet d'abord de pointer que l'engagement des élèves dans les tâches prescrites est une préoccupation des enseignants. Elle repose sur l'observation globale de la classe :

Melissa - Autoconfrontation simple (ACS) 2 - Tours de Parole (TP) 8 à 10 - Leçon de handball

Melissa : Tu vois, là, ça marche pas, ça ralentit...

Chercheur : Tu dis ça ...

Melissa : c'était trop dur, alors ça ralentit : Mais j'm'en rends compte tout de suite.

Les enseignants portent aussi une attention plus grande aux élèves qu'ils ont désignés au préalable au chercheur comme étant «à risque de décrochage ».

Hervé -ACS 1 - TP 34 - Leçon d'ultimate

Chercheur : ici, tu prends cet élève par le bras, ...Yohan, c'est ça ? 
Hervé : Oui, c'est Yohan... Lui, faut le surveiller comme le lait sur le feu, comme je te disais. Je le prends pour les démonstrations (...) Il peut te faire exploser la classe en deux minutes.

Les quatre enseignants sont également attentifs à l'intérêt de ce qu'ils proposent aux élèves. Ils disent que lors de certains cours, ils sont « sur le fil du rasoir ", que « tout peut basculer d'une seconde à l'autre » (Maude). Alors ils cherchent de façon continue à coconstruire du sens à propos des situations avec ces élèves au travers d'une activité masquée très importante. Ils procèdent par exemple à des formes de groupement fluctuantes qui font réussir tout le monde mais qui ne stigmatisent pas les plus faibles, ou à des interventions personnalisées (parfois publiques, parfois privées, pour valoriser, montrer une attention particulière ou au contraire rappeler à l'ordre).

\subsection{La gestion des dispenses en classe}

$31 \mathrm{Au}$ cours des 18 leçons analysées, le nombre des "dispenses d'EPS " varie de façon importante selon l'enseignant et la classe (de 0 sur 27 élèves à 8 sur 26 élèves). Précisément, dans les trois classes auprès desquelles nous avons recueilli des données avec l'enseignant 1 (anonyme), le nombre d'élèves absents et dispensés présents oscille entre 4 et 8 ; avec deux classes de l'enseignant 3 (anonyme), entre 0 et 2. La gestion de ces

Questions Vives, $N^{\circ} 25$ | 2016 
dispenses occupe l'activité des quatre enseignants de manière diverse. Certains évoquent à peine cette question et, en réponse aux questions du chercheur, il n'y a pas d'hésitation:

Melissa - Autoconfrontation simple (ACS) 1 -Tour de Parole (TP) 8 - Leçon d'acrosport

Chercheur : et cette élève-là, qu'est-ce qu'elle fait (à propos d'une élève dispensée présente)?

Melissa : Marion, elle sait qu'au moment des parades, elle doit aller aider un groupe. Et puis elle sait que je peux la questionner à tout moment... d'ailleurs plus loin, quand je les regroupe, je lui ai posé la question «Bon qu'est-ce qui est difficile aujourd'hui, Marion »? Tu te souviens?

Les enseignants Hervé et Maude ressentent à l'inverse un inconfort important en début de leçon lorsque des élèves se présentent avec des mots d'excuse ou des certificats médicaux. Les tensions émergent lors de dispenses ponctuelles sans mot d'excuse, ni certificat, ou lorsqu'il y a un grand nombre d'élèves non participants.

Hervé - ACS 2 - TP 15 à 17 - Leçon de course de demi-fond

Hervé : là aujourd'hui, je me retrouve avec une brochette d'éclopés. Du coup, je voulais faire quatre équipes...

Chercheur : d'éclopés?

Hervé : oui, d'éclopés ou de fatigués. Le résultat est le même. Le problème c'est qu'on ne peut jamais vraiment savoir si c'est une vraie entorse ou ... une entorse psychologique... un gros coup de mou ... certains ce sont des spécialistes.

Les enseignants sont tiraillés par l'impossibilité de prouver les raisons de l'absence. Ils identifient des élèves qui multiplient les absences ou les non participations :

Hervé - ACS 2 - TP 25 Leçon de course de demi-fond

Hervé : Samia, tu verras, elle a toujours un truc...

Maude - ACS 1 - TP 15 - Leçon de gymnastique

Nadia, elle en est à sa deuxième troisième,... après deux cinquièmes, elle en peut plus du collège (rire) ... elle fait partie des meubles (rire).

Quant à Christophe, il exprime davantage de doutes, il tend à faire confiance à l'élève. Au cours de plusieurs entretiens informels enregistrés, il reconnaît qu'il est difficile de distinguer une absence pour raison personnelle d'une véritable inaptitude physique mais aussi qu'une « raison personnelle » peut être « une bonne raison ».

Christophe - Entretien informel (EI) 3

Par principe, je fais confiance. Je me dis ça doit être dur pour lui \{élève Théo\}.

Pour les autres enseignants, la demande de ne pas participer à un cours est la source possible de conflits intrapsychiques car de nombreux cas de figures surviennent, les contraignant à des traitements personnalisés. Au cours de trois entretiens d'autoconfrontation, Maude et Hervé disent prendre des décisions parfois injustes (en renvoyer un en permanence et garder l'autre en classe, en réprimander publiquement un et ne faire aucune remarque à l'autre). De plus, la plupart de ces « cas » doivent être gérés dans l'urgence du début de leçon. On distingue d'abord les justifications d'absences antérieures. L'élève présente un certificat médical ou, selon les établissements une « autorisation du CPE », voire un « mot des parents » à la place ou en complément. Malgré la pression temporelle, les quatre enseignants interrogent toujours l'élève lorsqu'ils ne connaissent pas la raison de l'absence. Au-delà de la dimension officielle de la dispense, de l'autorité médicale ou parentale qui la délivre, il s'agit pour eux de faire un diagnostic sous pression temporelle : 

préalable filtrées par le CPE. Autrement dit, un élève qui demande à ne pas participer "parce qu'il s'est tordu la cheville en descendant du car» ou qui présente une justification d'absence de la part de ses parents ou d'un médecin doit d'abord voir le CPE avant de se présenter à l'enseignant d'EPS. De plus, il se présente en cours avec une décision ou une suggestion du CPE. Cette situation réduit d'autant les négociations directes menées par l'enseignant. A l'inverse, Maude est préoccupée par le fait que, selon elle, le CPE responsable des classes de troisième dans son collège échange peu et sur un mode souvent impatient, parfois agressif.

Maude - ACS2 - TP123

Maude : le CPE, M.X, y montre bien que nos soucis d'EPS, c'est pas son problème

Chercheur : Et ça pose des problèmes ? $\mathrm{Ca}$...

Maude : J'évite de le rencontrer. Bon, les élèves aussi, remarque, ça c'est bien (rire).

Chercheur : non mais ça ...

Maude : ben, tout est plus compliqué... pour contacter les parents. Ou alors, l'autre jour j'avais une sortie en CO \{course d'orientation\}, ben j'avais des dispensés que je garde d'habitude... j'pouvais pas les prendre et les garder dans le froid pour des heures sans rien faire. Ça a été toute une histoire pour les mettre en permanence \{lieu de garde des élèves par les CPE\}. Il m'fait bien comprendre que je l'emm... 
Maude énonce aussi que cette collaboration défaillante a un effet sur son "assurance " face aux élèves en cas de problème ponctuel ou récurrent. Autrement dit, le fait de se sentir souvent en porte-à-faux avec le CPE la prive de certaines sanctions (comme le renvoi d'un élève en permanence), ce qui rend les négociations plus difficiles face aux élèves et accentue les conflits intrapsychiques dans son activité.

Le croisement des données met en évidence la même variation du degré de collaboration avec les Professeurs Principaux des classes qui sont pour certains des interlocuteurs privilégiés et disponibles, qui recoupent les informations à propos de tel élève, prennent l'initiative de convoquer les parents, témoignent de l'intérêt pour la question des absences en EPS. Melissa évoque au contraire que certains Professeurs Principaux sont « invisibles» ou «ne font pas grand-chose». Les participants à l'étude sont tous les quatre également Professeurs Principaux de classe et rapportent leur jugement à leur propre activité dans ce domaine.

Les parents sont les autres adultes directement impliqués dans la gestion des dispenses en EPS. D'une part, dans deux des trois collèges de l'étude, ils ont l'opportunité " d'excuser " leur enfant pour une non participation. Il s'agit d'une tolérance qui ne donne pas lieu à une prescription formelle mais plutôt un usage local. Dans ces cas, les négociations de l'élève avec l'enseignant peuvent déborder le cadre de la classe et donner lieu à un traitement particulier de l'élève (partir en permanence, rester en classe avec ou sans tâche à réaliser). Ces mots d'excuses peuvent aussi être sujets à caution aux yeux des enseignants. Dès lors, les raisons invoquées par les parents pour justifier une absence de participation impliquent souvent une sorte d'enquête de la part de l'enseignant ou une prise de contact direct avec eux.

Melissa - Hervé - ACC 1 - TP 79 Leçon de course de demi-fond

He : l'année dernière, j'ai eu un mot. Les parents y voulaient pas que leur gosse fasse du basket. « Mon frère s'est cassé le nez au basket. Alors ma mère elle veut pas que je fasse basket. » Alors là, il faut discuter avec les parents, ...

\subsection{L'activité conjointe entre enseignants d'EPS}

51 L'activité de chacun des quatre participants à l'étude pour prévenir les dispenses des élèves dépend également en grande partie des accords entre enseignants d'EPS sur ce sujet. Ainsi, dans les trois établissements de l'étude, nous avons pu analyser des formes de prévention du décrochage scolaire en EPS diverses: dans un des trois établissements (celui de Melissa), les négociations professeurs-élèves sont opérées sur la base de décisions communes à tous les enseignants et affichées (prévention concertée): le principe est de garder en classe d'EPS l'élève ayant un mot ou un certificat et de lui donner systématiquement des tâches à réaliser: arbitrage, parade, co-évaluation. Les enseignants de ce collège avaient à disposition des exemples de tâches qu'ils avaient mises en commun pour réagir immédiatement lorsque le cas se présentait. Au moment de l'étude, un dispositif « d'EPS adaptée » pour les élèves dispensés de longue durée était en cours d'élaboration mais butait sur des questions de moyens en personnel et de créneaux horaires. C'est également le collège où les observations mettent en évidence que le nombre de dispensés est le plus faible.

52 Cela est corrélé, dans la classe de Melissa, avec des consignes argumentées à propos de ces activités alternatives. Comme auprès des élèves non dispensés, l'enseignante prend à son compte à ce niveau les préoccupations de prévention du DS par une construction du 
« sens » de cette EPS adaptée, en même temps que la recherche de « réussite » des élèves dispensés.

Dans les deux autres, les formes de prévention sont menées individuellement par les enseignants (prévention juxtaposée ou contradictoire). Par exemple, Maude et Christophe travaillant dans le même établissement conduisent des négociations sensiblement différentes avec les élèves présentant des mots d'excuses et des certificats médicaux, l'un compréhensif et peu exigeant avec les élèves, privilégiant les interactions privées avec l'élève concerné, l'autre négociant de façon plus serrée, demandant des justifications, privilégiant les interactions publiques avec l'élève concerné, menaçant d'envoyer en permanence ou au contraire de garder en cours selon les besoins.

\section{Discussion}

Dans cette étude de type clinique, bien que le DS en EPS du côté des élèves prenne des formes spécifiques (dispenses, interruptions de l'activité pour des fatigues ou douleurs parfois simulées), les modalités de prévention de la part des enseignants, telles qu'elles apparaissent à l'issue du traitement des données, semblent comporter de nombreux points communs avec celle des enseignants des autres disciplines. Ce point a déjà été mis en évidence par d'autres auteurs (Méard, 2013 ; Bergamaschi, 1014). On repère la double nécessité pour l'enseignant de "donner du sens » en faisant jouer, en expliquant les motifs du travail prescrit et «de rendre efficient » en évitant notamment les situations d'échecs répétés. La volatilité et l'imprévisibilité des modes d'engagement d'élèves en classe d'EPS, décrit en milieu difficile par exemple par Vors et GalPetitfaux (2009), fait donc porter la persistance de l'engagement sur les situations elles-mêmes, offrant cette double opportunité (donner du sens et rendre efficients). La part masquée de l'activité des élèves, déjà pointée par Guérin, Pasco \& Riff (2008), se manifeste par des formes de désengagement subtiles, dont la demande de dispense n'est qu'un aspect. Elle se manifeste aussi par des modes de détournement des tâches prescrites par l'enseignant, selon des modalités proches de celles décrites par Saury \& Rossard (2009). Si l'on considère l'élève comme un être humain au travail, ces résultats convergent avec ceux de l'ergonomie francophone depuis 50 ans (Daniellou, 1996).

Un des points saillants de l'étude touche la confusion entre les préoccupations des enseignants vis-à-vis de l'engagement des élèves et celles relatives à la prévention des comportements perturbateurs d'élèves. Avec les outils de l'analyse didactique, d'autres travaux font un constat comparable, notamment chez les enseignants travaillant en milieu difficile (Poggi \& Brière-Guenoun, 2014). En fait, on peut avancer que l'engagement des élèves est souvent au service du contrôle de classe, ce qui entraîne des conflits intrapsychiques: stimuler ou calmer la classe, faire apprendre (au risque de rendre le climat trop studieux) ou distraire (au risque de provoquer de l'agitation). Ces conflits intrapsychiques concernent prioritairement les moments de décrochage des élèves garçons qui peuvent devenir source de perturbations, de bruit, des activités parallèles à celle du cours. En revanche, l'abandon de certaines collégiennes est moins préoccupant. Il passe davantage inaperçu, ce qui n'est pas sans interroger. En effet, on peut se demander si le discours sur la prévention du décrochage ne cache pas finalement une préoccupation centrale de gestion du désordre dans l'activité enseignante et si celle-ci ne prend pas systématiquement le pas sur la priorité réelle accordée à la participation active des élèves, notamment des filles. Ce résultat illustre, dans ce contexte particulier de 
l'enseignement de l'EPS, le processus de construction des inégalités scolaires tel qu'il a été repéré par plusieurs sociologues (Rochex \& Crinon, 2011).

La gestion du phénomène spécifique de la dispense occupe une place assez importante dans l'activité des enseignants d'EPS de l'étude. Trois d'entre eux sont traversés de conflits intrapsychiques qui prolongent et aiguisent les autres transactions en cours de leçon. Les négociations en début de leçon, publiques et dans l'urgence les placent parfois dans des situations d'inconfort.

Elles impliquent le développement de partenariats éducatifs, notamment avec les parents et les autres personnels éducatifs, comme l'ont déjà montré explicitement Tièche Christinat et Gilles (2013). Dès lors, l'étude met en évidence que la décision qui fait suite à la justification plus ou moins fondée d'une absence d'élève est distribuée selon une organisation multipolaire (knotworking). Et la classe n'apparaît plus comme un environnement exclusivement organisé selon un modèle hiérarchique où l'enseignant est seul responsable des faits qui s'y déroulent. L'analyse débouche plutôt sur la description d'une organisation émergeante et autonome, d'un centre de décision résultant de l'activité conjointe de l'enseignant, des élèves et des partenaires et où chacun cherche à déborder sur les prérogatives de l'autre (Engeström, 2000).

Ces processus de knotworking et de boundary crossing créent des tensions dans les systèmes d'activité. On les observe dans les dispositifs spécifiques de lutte contre le DS engageant plusieurs acteurs (enseignants, éducateurs, partenaires éducatifs). De tels dispositifs ont été étudiés par Flavier, Moussay et Méard (2015) en collège. Ils rassemblent les différents acteurs de la communauté éducative élargie de l'établissement, dont certains comme l'assistante sociale, l'infirmière scolaire ou la conseillère d'orientation psychologue.

On se rend compte aussi que les décisions collectives d'enseignants d'EPS, le fait qu'ils mettent en place un "règlement des dispenses", permettent de peser dans ces partenariats en présentant aux élèves, parents et infirmières les termes d'un contrat consistant et des postures assurées. En ce sens, les formes de prévention du décrochage scolaire en EPS apparaissent d'un certain point de vue comme le signe de la vitalité ou de l'absence de vitalité des collectifs de travail dans les établissements.

Dans la prévention du DS, en EPS comme ailleurs, la complexité tient essentiellement à la simultanéité des préoccupations et la distribution de la décision, impliquant dès lors un traitement global mais également individualisé, souvent sous pression temporelle, du phénomène au regard de la singularité des situations rencontrées. Ainsi, le processus de DS ne peut plus être appréhendé uniquement comme un processus identifiable à partir de tendances ou de caractéristiques générales, mais au contraire dans ce qu'il a de particulier en référence à l'idiosyncrasie des situations et au sens qu'elles revêtent pour les acteurs en lien avec leurs autres expériences.

61 Si le moment de la classe reste le lieu des imprévus et constitue sans aucun doute la partie la plus instable du travail de l'enseignant, les contraintes qui s'exercent sur le métier d'enseignant (effectifs nombreux d'élèves, environnement difficile, complexité, diversité des publics réunis dans une même classe, etc.) sont autant de facteurs de variation de l'engagement de l'enseignant lui-même (Mukamurera \& Bouthiette, 2008). Plus particulièrement, les élèves à risque de DS posent des défis quotidiens majeurs aux enseignants qui ne sont pas toujours en mesure de les relever. Le travail de l'enseignant impacte le processus du DS de l'élève, mais ce décrochage de l'apprenant confronte en retour l'enseignant à des contraintes fortes, caractérisées par l'émergence de situations 
d'empêchement ou de renoncement, mettant à mal la réalisation de son métier (Clot, 2008). Le caractère interpénétré des deux modes d'engagement, celui de l'adulte et celui de l'élève, résiderait dans les tensions repérables entre les motifs et les opérations respectives de l'un et de l'autre.

\section{Conclusion}

62 Avant de réaliser cette étude, nous avions envisagé de fonder notre démarche clinique sur les chiffres des absences, dispenses déclarées en cours et aux examens du secondaire où l'EPS est obligatoire. Après divers contacts et des recherches de collaborations réitérées avec les instances académiques dans laquelle s'est déroulée cette étude, nous avons pu accéder aux seuls chiffres concernant les inaptes au baccalauréat, ce qui en dit beaucoup sur le fait que, dans un contexte où la question du DS est omniprésent, celle des absences et abandons en EPS n'est pas une priorité.

63 La "dispense » d'EPS reposant sur une inaptitude simulée apparaît donc comme un épiphénomène. On peut penser à l'inverse que c'est un type de décrochage lourd de conséquences sur la santé et la sécurité des élèves à long terme. Il s'agit aussi d'une démonstration de non-citoyenneté et d'un aveu d'impuissance des acteurs de l'institution scolaire face à une infraction connue de tous (et parfois source de fierté pour l'élève). Pour aller à l'essentiel, c'est à ce moment de sa formation que le jeune apprend de sa propre expérience ou de l'observation de certains de ses pairs qu'on peut, avec la complicité de l'autorité médicale et parentale, «se faire porter malade » dans son travail.

64 À ce stade, nous ne pouvons formuler qu'une seule préconisation en direction des professionnels: la prévention du DS en EPS implique de renouveler des pratiques d'enseignement pour que l'élève surmonte ses difficultés d'apprentissage, investisse le travail scolaire de sens et s'adapte à un système réglementaire consistant et partagé par les adultes. Le seul véritable moyen de lutter contre l'abus de la « dispense d'EPS » est, au niveau de la classe comme au niveau de l'établissement, de revitaliser des collectifs de travail.

65 Cette étude s'ouvre de notre point de vue vers deux perspectives : d'abord, sans lâcher les présupposés de l'analyse d'activité, il nous semblerait heuristique d'éclairer les dynamiques d'engagement en classe par une analyse didactique plus serrée des « types de situations ». Cela permettrait de mettre en regard l'activité des élèves et de l'enseignant avec les formes de mise à l'étude, les modalités d'intervention et de régulation de l'enseignant. Enfin, ce travail pourrait être heureusement complété par un questionnement relatif aux corrélations éventuelles entre le décrochage global et le décrochage en EPS. Cela requiert une démarche quantitative auprès d'un échantillon beaucoup plus nombreux que le nôtre. Et il serait intéressant, selon les résultats obtenus, soit de chercher une corrélation (ce qui permettrait de fonder des stratégies précoces de dépistage du DS et de prévention par un traitement collectif de la dispense d'EPS), soit de constater à l'inverse que les élèves qui se marginalisent de l'institution ne sont pas ceux qui manquent l'EPS (ce qui permettrait d'envisager des dispositifs utilisant le levier de cette discipline pour récupérer des jeunes "qui s'éloignent de l'école ", dispositifs que l'on perçoit d'ailleurs ici ou là et qui empiriquement augmentent le nombre d'heures d'EPS pour des élèves à haut risque de décrochage). 


\section{BIBLIOGRAPHIE}

Bautier, E. (eds) (2002). Décrochage scolaire : genèse et logique des parcours. Rapport de recherche pour la DPD / MEN.

Bélanger, J., Bowen, F., Cartier, S., Desbiens, N., Montésinos-Gelet, I., \& Turcotte, L. (2012). L'appropriation de nouvelles pratiques d'interventions pédagogiques et éducatives en milieu scolaire : réflexions sur un cadre théorique intégrateur. Éducation et francophonie, 40(1), 56-75.

Bergamaschi, A. (2014). Le processus d'accrochage-décrochage scolaire au prisme de la dispense en EPS. In E. Flavier \& S. Moussay (eds). Répondre au décrochage scolaire. Expériences de terrain. Bruxelles : De Boeck.

Bergamaschi, A. \& Méard, J. (2012). Sport and School Integration: a New Approach to Understand the Dropout in Junior High Schools. Theoretical Proposal Applied to French Case, Journal of Physical Education and Sport, 12(3), 267 - 273.

Bernard, P-Y. (2011), Le décrochage scolaire. Paris, PUF.

Blaya, C. (2010). Décrochages scolaires : l'école en difficulté. Bruxelles : De Boeck.

Blaya, C. (2013). Le décrochage scolaire dans les pays de l'OCDE. Regards croisés sur l'économie, 2, 69-80.

Blaya, C., \& Hayden, C. (2003). Constructions sociales et décrochages scolaires et absentéismes en France et en Angleterre. LARSEF/Observatoire européen de la violence scolaire.

Bonnery, S. (2007). Comprendre l'échec scolaire. Élèves en difficultés et dispositifs scolaires. Paris, France: La Dispute.

Brown, B.-A. (2010). Social Hostility and the "Dropout" Syndrome: Leadership Assisting Youths' Re-Entry into School? Educational Review, 62(1), 53-67.

Brown, T.M. \& Rodriguez, L.F. (2009). School and the Co-Construction of Dropout. International Journal of Qualitative Studies in Education, 22(2), p. 221-242.

Bruno, F. (2015). Analyse du développement du pouvoir d'agir d'enseignants confrontés au risque de décrochage scolaire : étude de cas en classe de sixième. Thèse de doctorat, Aix-Marseille Université, non publiée.

Bruno, F., Méard, J. \& Walter, E. (2014). Les dispositifs français de lutte contre le décrochage scolaire en collège : ce qui est prescrit et ce qui est mis en œuvre. Orientation scolaire et professionnelle, 42/4. http://osp.revues.org/4183; dOI : 10.4000/osp.4183

Cairns, R., Cairns, B., \& Neckerman, H. (1989). Early school dropout: Configurations and determinants. Child Development, 60, 1437-1452.

Clot, Y. (2008). Travail et pouvoir d'agir. Paris, France : PUF.

Commission Européenne (2000). European report on quality of school education. Sixteen quality indicators. (Rapport)

Daniellou, F. (Dir.)(1996). L'ergonomie en quête de ses principes. Débats épistémologiques. Toulouse : Octarès. 
Deslandes, R., \& Lafortune, L. (2001). La collaboration école-famille dans l'apprentissage des mathématiques selon la perception des adolescents. Revue des Sciences de l'Éducation, 27(3), 649669.

Durand, M. (1996). L'enseignement en milieu scolaire. Paris: PUF.

Dusenbury, L., Brannigan, R., Hansen, W., Walsh, J., \& Falco, M. (2005). Quality of implementation: developing measures crucial to understanding the diffusion of preventive interventions. Health Education Research, 20(3), 308-313.

Engeström Y (2000). Activity theory as a framework for analyzing and redesigning work. Ergonomics, 43(7), 960-974.

Engeström, Y. (2004). New forms of learning in co-configuration work. Journal of Workplace Learning, 16, 11-21.

Félix, C., \& Saujat, F. (2008). L'aide au travail personnel des élèves entre déficit de prescriptions et « savoirs méthodologiques » : un double regard didactique et ergonomique, Dossiers des Sciences de l'Éducation, 20, 123-136.

Flavier, E., \& Moussay, S. (2014). Répondre au décrochage scolaire. Expériences de terrain. Bruxelles, (B) : De Boeck.

Flavier, E., Moussay, S., \& Méard, J. (2015). Les initiatives individuelles au cœur des dispositifs collectifs locaux de prévention du décrochage scolaire. Les dossiers des sciences de l'éducation, 33, 175-192.

Guérin, J., Pasco, D., \& Riff, J. (2008). Activités dissimulée et publique d'un élève décrocheur en mathématiques. Les Sciences de l'éducation pour l'ère nouvelle 1/2008 (41), 11-31.

Houlfort, N., \& Sauvé, F. (2010). Santé psychologique des enseignants de la Fédération autonome de l'enseignement. Montréal, Québec : École nationale d'administration publique.

Karsenti, T., \& Collin, S. (2009). L'autre décrochage scolaire. Formation et Profession, 16 (1), 2-6.

Lee, V.E., \& Burkam, D.T. (2003). Dropping out of high school: The role of school organization and structure. American Educational Research Journal, 40(2), 353-393.

Leontiev, A.N. (1984). Activité, conscience, personnalité. Paris, France : Éditions du Progrès. Lessard, A., Fortin, L., Royer, E., Potvin, P., Marcotte, D., \& Joly, J. (2007). Cheminements de décrocheurs et de décrocheuses. Revue des sciences de l'éducation. 33(3), 646-662.

Méard, J. (2013). L'activité des élèves en risque de décrochage scolaire en EPS : la dynamique des interactions en classe. eJRIEPS, 30, 99-104.

Méard, J. (2014). La co-construction de sens dans les interactions entre l'enseignant et les élèves à risque de décrochage. In E. Flavier \& S. Moussay (eds.). Répondre au décrochage scolaire. Expériences de terrain (pp. 39-50). Bruxelles (B) : De Boeck.

Méard, J., Bertone, S., \& Flavier, E. (2008). How fourth grade-students internalize rules during teacher-student(s) transactions. A case study. British Journal of Educational Psychology, 78, 395-410.

Mottet, P., Meraite, A.A., \& Cloes, M. (2007). Solutions imaginées par les enseignantes en éducation physique du secondaire pour lutter contre le phénomène de la dispense dans le cours de natation. eJRIEPS, 11, 106-.119.

Mukamurera, J., \& Bouthiette, M. (2008). Rester dans l'enseignement ou quitter ? Portrait de la situation et motivation des enseignants. Séminaire « Pourquoi et comment soutenir l'insertion 
professionnelle de nouveaux enseignants au Québec ? Résultats de recherche et pistes d'action. ». Québec : Université de Sherbrooke.

Poggi, M. P., \& Brière-Guenoun, F. (2014). Ce qui s'enseigne en milieu difficile : tentative d'articulation des approches sociologique et didactique. In N. Wallian, M-P. Poggi \& A. ChauvinVileno (Eds.), Action, interaction et intervention : à la croisée du langage, de la pratique et des savoirs (pp. 33-344). Berne : Peter Lang.

Rochex, J-Y., \& Crinon, J. (2011). La construction des inégalités scolaires. Rennes: PUR.

Rumberger, R. (2011). Dropping out. Why students drop out of high school and what can be done about it. Cambridge (Ma) : Harvard University Press.

Saury, J., \& Rossard, C. (2009). Les préoccupations des élèves durant des tâches d'apprentissage coopératives et compétitives en badminton : une étude de cas. Revue des Sciences de l'Éducation, 35 (3), 195-216.

Sensevy, G., \& Mercier, A. (2007). Agir Ensemble. L'action didactique conjointe du professeur et des élèves dans la classe. Rennes : Presses Universitaires de Rennes.

Thibert, R. (2013). Le décrochage scolaire : diversité des approches, diversité des dispositifs. Dossier d'actualité, Veille et Analyses IFE, 84. Lyon, France : ENS de Lyon.

Tièche Christinat, C. \& Gilles, J.-L. (2013). Alliances éducatives et accrochage scolaire. Education \& Formation, 300, 9-14.

Thijs, J., Verkuyten, M., \& Helmond, P. (2010). A further examination of the big-fish-little-pond effect: perceived position in class, class size, and gender comparisons. Sociology of Education, 83(4), 333-345.

Thornberg, R. (2010). School democratic meetings: Pupil control discourse in disguise. Teaching and Teacher Education, 26, 924-932.

Vors, O., \& Gal-Petitfaux, N. (2009). Construire une activité collective de travail dans une classe d'EPS en "Réseau ambition réussite" : entre masquage et ostentation. eJRIEPS, 18, 156-177.

Vygotski, L.S. (1934/1997). Pensée et langage. Paris : La Dispute.

\section{RÉSUMÉS}

Le décrochage scolaire en EPS se traduit par des absences perlées ou à répétition qui reposent souvent sur des déclarations d'inaptitudes simulées ou non (" dispenses »). Ces absences plutôt féminines bénéficient souvent de complaisances de la part des adultes: parents, infirmière scolaire, médecins. Dans le cadre d'un programme de recherche, nous avons cherché à comprendre les éventuelles spécificités du décrochage en EPS en analysant l'activité de quatre enseignants de collège pendant 18 leçons, par le biais de méthodes directes (observations, entretiens semi-directifs, entretiens informels) et indirectes (instructions au sosie, entretiens d'auto-confrontation). L'étude montre que l'activité des enseignants en classe porte des similitudes avec celles des autres enseignants confrontés au décrochage. Concernant la gestion des « dispenses d'EPS », les négociations professeurs-élèves peuvent être âpres et se fondent sur des décisions communes à tous les enseignants et affichées ou menées individuellement. De là, les formes de prévention du décrochage scolaire en EPS apparaissent comme le signe de la vitalité ou de l'absence de vitalité des collectifs de travail.

The signs of School dropout in Physical Education (PE) are beaded or repeated absences often based on statements simulated or not unsuited "exemptions". These rather feminine absences 
often benefit from complacency on behalf of adults: parents, school nurses, doctors. As part of a research program, we tried to understand the possible PE dropout prevention characteristics by analyzing the activity of four college teachers for 18 lessons, through direct method tools (observations, semi-structured interviews, informal interviews) and indirect method tools ("instructions to the double", self-confrontation interviews). The study shows that the activity of the classroom teacher bears similarities with other teachers as regard of dropout prevention. Concerning the management of "PE exemptions" negotiations between students and teachers can be tough and based on joint decisions to all teachers and displayed or taken individually. From there, the forms of dropout prevention in PE appear as a sign of the vitality or lack of vitality of collective labor among PE teachers.

INDEX

Keywords : décrochage scolaire, enseignement, clinique de l'activité, développement professionnel, collectif de travail

\section{AUTEUR}

\section{JACQUES MÉARD}

Equipe LASALé, Haute École Pédagogique, Canton de Vaud, Lausanne (CH) 